\title{
Evaluation of clinical benefits achievable by using different optimization algorithms during real-time prostate brachytherapy
}

\author{
Marta Adamczyk a,*, Grzegorz Zwierzchowski a,b, Julian Malicki a,b, \\ Janusz Skowronek ${ }^{c}$
}

\author{
${ }^{a}$ Department of Medical Physics, Greater Poland Cancer Centre, 15 Garbary St., 61-866 Poznan, Poland \\ ${ }^{\mathrm{b}}$ Electroradiology Department, University of Medical Sciences, Poznan, Poland \\ 'Brachytherapy Department, Greater Poland Cancer Centre, Poznan, Poland
}

Received 16 November 2010; received in revised form 9 December 2011; accepted 16 December 2011

Available online 4 January 2012

\section{KEYWORDS \\ Prostate cancer; \\ Brachytherapy; \\ High dose rate}

\begin{abstract}
Background and purpose: High dose rate (HDR) brachytherapy is a clinically used procedure in prostate cancer treatment. The purpose of this study was to present the influence of using different optimization algorithms in 3D-CBRT planning on the treatment plan quality. Materials and methods: Treatment plans were calculated for 15 patients - three plans for each patient using: geometrical optimization (GO), inverse optimization (IO) and blind inverse optimization (BIO). For each patient, PTV and OAR volumes, number of needles and geometry of the implant were set equal. Differences between dose distributions were tracked using: D90, V100, V200, Dmax (for prostate); D10, Dmax (for urethra); D10, V100, Dmax (for rectum). Results: The analysis of mean values of $\mathrm{D} 90$ and $\mathrm{V} 100$ in the prostate showed that inverse algorithms gave the best results (mean D90 was $12.1 \%$ for $\mathrm{BIO}$ and $9.3 \%$ for $\mathrm{IO}$ better than for $\mathrm{GO}$, mean $\mathrm{V} 100$ was $8.2 \%$ for $\mathrm{BIO}$ and $6.3 \%$ for $\mathrm{IO}$ better than for $\mathrm{GO}$ ). From a clinical point of view, GO diminished the doses in the PTV and urethra in all analyzed parameters. The lowest mean doses in the rectum were achieved for plans optimized with $\mathrm{IO}$ and BIO (mean D10: 61.2\% for GO, $58.1 \%$ for IO, $58.0 \%$ for BIO; mean Dmax: $92.8 \%$ for GO, $85.1 \%$ for IO, $83.6 \%$ for BIO).

Conclusions: Application of the blind inverse optimization ( $\mathrm{BIO}$ ) algorithm led to clinically best dose parameters for PTV and the rectum. Use of geometrical optimization (GO) led to smaller doses in the urethra, which was however associated with a certain dose decrease also in PTV. (c) 2011 Associazione Italiana di Fisica Medica. Published by Elsevier Ltd. All rights reserved.
\end{abstract}

\footnotetext{
* Corresponding author. Tel.: +48 618850553; fax: +48 618850551.

E-mail address: marta.adamczyk@wco.pl (M. Adamczyk).
} 


\section{Introduction}

The number of patients, among other patients with prostate cancer, treated using external beam radiotherapy (EBRT) combined with high dose rate brachytherapy (HDRBT) iridium-192 stepping source implants is increasing rapidly [1-4]. Different fractionation schemes (doses, number of EBRT/HDR-BT fractions) are used and reported in the literature for this combined schedule of prostate treatment [5-7]. Generally, patients receive EBRT as 40-50 Gy in 20-25 fractions over four or five weeks, which is followed by a temporary brachytherapy implant. The second part of this clinically used treatment schedule is defined as transrectal ultrasound (TRUS) guided prostate brachytherapy [8] using the iridium-192 remote afterloading technique. In the Brachytherapy Department of the Greater Poland Cancer Centre, this kind of 3D-conformal real-time HDR brachytherapy (3D-CBRT) is applied mostly as a boost (in one or two fractions, giving 10-15 Gy per fraction) [9].

The use of this combined treatment scheme is possible by developing theoretical and experimental studies, brachytherapy equipment and facilities, computer devices with treatment planning software, and, of course, clinical experience [10]. Consequently, all advanced technology available for iridium-192 brachytherapy has made anatomyrelated dose optimization possible [11]. By using different optimization algorithms, the treatment planning system (TPS) calculates the dwell times and dwell positions of the source for and along each catheter $[9,12,13]$. Modulation of the radiation intensity by adaptation of dwell times and positions of the source along the catheters provides the appropriate dose distribution for the PTV (in terms of target coverage and dose homogeneity), with lower doses to organs at risk (OARs: urethra and rectum) $[5,7,14,15]$. This is the part of the optimization process during which various calculation algorithms implemented in the TPS are used $[9,15]$.

Using all these modalities, pre-treatment and treatment investigations with implant procedures (including dose and fractionation) are still under ongoing clinical research. This study is part of the investigation of possible benefits from using different algorithms for optimizing dose distribution.

\section{Aim}

The aim of this study was to investigate the influence of using different optimization algorithms in real-time treatment planning of 3D-CBRT on the treatment plan quality by analyzing dose distribution in the prostate gland (PTV) and organs at risk (OARs: urethra, rectum).

\section{Materials and methods}

45 treatment plans for 15 consecutive patients treated in the Greater Poland Cancer Centre were prepared in Oncentra Prostate ${ }^{\circledR}$ version 3.0.9 (Nucletron B.V., Veenendaal, the Netherlands), a TPS dedicated to real-time prostate brachytherapy treatment planning. In every case the prescribed dose was $10 \mathrm{~Gy}$. The whole planning procedure, including needle implantation and preparation of treatment plan, was based on transrectal ultrasound (TRUS) images $[8,12,16,17]$. A microSelectron HDR (Nucletron B.V., Veenendaal, the Netherlands) device with iridium-192 source was used to deliver the dose $[1,3,4,18]$. Implantation of needles, treatment planning procedure and irradiation were carried out under extradural or general anaesthesia in the HDR treatment room [14,19].

During treatment planning procedure, the clinical target volume (CTV) was represented by the whole prostate gland visible on $1 \mathrm{~mm}$ separated TRUS images [5]. If there is any risk of involvement, the medial part of the seminal vesicles can be included in the CTV [20]. In our clinical practice with the real-time intraoperative planning, no margin is applied and thus the planning target volume (PTV) is considered to be CTV $[7,17,20,21]$, resulting in smaller PTV volumes than for external beam radiotherapy [22].

First, the procedure of pre-planning was done. On images acquired by a TRUS device, CTV/PTV and OARs (urethra and rectum) were delineated [21]. Then the geometry of the implant was designed (the number and positions of virtual needles) [16]. After implanting nonactive needles, contours were evaluated and the geometry of the implant was reconstructed on a second set of ultrasound images. Based on 'actual' geometry, a treatment plan was generated. Except routine dose-volume histogram (DVH) analysis, the quality of the plan was evaluated taking into account some additional parameters: D90, V100, V200, Dmax (for prostate); D10, Dmax (for urethra); D10, V100, Dmax (for rectum) [5,17,23-26]. To evaluate conformity of generated plans, the conformal index (COIN) was calculated according to the formula $[15,23,24,26]$ :

$\mathrm{COIN}=c_{1} c_{2}$

with:

$c_{1}=\frac{\text { PTV }_{\text {ref }}}{\text { PTV }}$

$c_{2}=\frac{\text { PTV }_{\text {ref }}}{V_{\text {ref }}}$

where: PTV - the planning target volume, PTV ref $_{-}$the volume of PTV covered by the prescribed dose, $V_{\text {ref }}-$ the volume covered by the prescribed dose.

All these indices were used to evaluate the influence of different optimization algorithms on final plan's quality, as optimization algorithms implemented in TPS give the opportunity to adapt the dwell times and dwell weights at the dwell positions of the source in each available catheter. Oncentra Prostate ${ }^{\circledR}$ offers five groups of optimization methods. Two of them - manual adjustment and graphical optimization - were not considered because of their subjective character during the dose distribution calculation. Manual adjustment is based on manual definition of dwell weights or times, while in graphical optimization the change of these dwell weights or times is a consequence of changing the shape of the isodose line. That is why, finally, three optimization algorithms were verified in the study: geometrical optimization (GO), inverse optimization (IO) and blind inverse optimization (BIO).

Geometrical optimization (GO) represents an a priori method of optimizing. Adjusting the dwell weights at the 
source dwell position according to the density of the neighbourhood of each dwell position, is done by homogenizing the dose distribution around the catheters. This tool is equipped with a volume option and a distance option. The first one is used to avoid cold areas between catheters. The distance method gives a more homogeneous dose distribution when a small number of catheters is used or their geometry is highly irregular [27]. In this study the possibilities of the volume option were analyzed.

Using single solution inverse optimization (IO) (with two methods to select: variance-based inverse optimization (SVBO) or DVH-based inverse optimization (SDVHO)) requires user's preferences to be expressed. After defining PTV, OARs and catheters' positions, the user determines the criteria of dose distribution quality to be considered. This 'ideal' dose distribution demands are transformed into 'real' dose distribution quality by estimating dwell weights and dwell times within available catheters [27]. In this study, plans calculated using inverse algorithm were optimized in the SVBO mode. The urethra was set as a volume of interest with higher priority than the prostate. The density of sampling points on closed PTV volume equals 7.0 points per $\mathrm{cm}^{2}$, while the importance factors (weights) were: 0.500 for prostate-conformity and 0.250 for its homogeneity. Urethra's and rectum's importance factors equal 0.250 and 0.001 , respectively with dose limits of $120 \%$ for the urethra and $85 \%$ for the rectum. Additionally, for the rectum, the 5-mm surface margin was created to produce dose sampling points both on the surface of this OAR and in the $5-\mathrm{mm}$ neighbourhood of the PTV. The dwell time gradient restriction, which can be chosen from 0.0 to 1.0 , that is from ignoring dwell time gradient objective to its maximum consideration, equals 0.2 . From convergence settings, high accuracy was chosen with 1000 as maximum iterations number. This improves the precision for the convergence of the algorithm, while increasing the execution time.

Multi-solution blind inverse optimization (BIO), with three methods: variance-based blind inverse optimization (MVBO), DVH-based blind inverse optimization (MDVHO) and DVH-based optimization using evolutionary algorithms (MDVHOE), represents an optimization method which provides a set of potential solutions to the problem from the pareto frontier optimal set [26]. The objective function of this algorithm eliminates the problem of having to know the most appropriate values of important factors for PTV and OARs. Here multiple runs of the optimization engine are done to find many solutions that can be handled by the system. To conclude, this tool works in two steps. The first one is optimization, when the system offers different alternatives which correspond to patient's individual anatomy. The second one concerns the decision procedure during which the most appropriate alternative is selected [27]. Plans generated for the purpose of this study were calculated using the MVBO mode. Priorities, density of sampling points, urethra's and rectum's dose limits, rectum's surface margin, dwell time gradient restriction and convergence settings used in the study were the same as for 10 plans. The importance factor was re-built into two parameters: 'importance from' and 'importance to'. For every structure (prostate-conformity, prostatehomogeneity, urethra and rectum), 'importance from' was set at 0.001 and 'importance to' was set at 1.000 with a focus parameter used to prostate-conformity, as multifocus is not allowed. The additional parameter, which was set as 4.0 , is the number of steps per importance factor. Its value determines the process of sampling the pareto front, which has the influence on the total number of solutions.

Independently of the optimization algorithm used, in our clinical practice each prepared plan is accepted when dose delivered to $10 \%$ of the urethra is under $135 \%$ of the prescribed dose. The dose to the anterior surface of the rectal mucosa should be under $100 \%$ of the prescribed dose [21]. That is why, when BIO algorithm was used, we try to choose plans fulfilling OARs' dose criteria. In the case of three patients D10 for the urethra was slightly higher (135.72\%, $137.35 \%$ and $137.47 \%)$, while the values of therapeutic indices achieved for those patients (D90, V100) were significantly higher. For two other patients, the values of maximum doses delivered to the rectum equalled $112.8 \%$ and $148.3 \%$, also at the cost of better values of D90 and V100. Concluding, the values of parameters for OARs which are over limits are analyzed and can be accepted taking into account the relation between the dose delivered and OARs' volumes (dose delivery to less than $1 \%$ of each organ volume). The values of the urethra and rectum indices are also analyzed individually taking into account the individual patients' characteristics (pubic arc interference, oblique course of the urethra or post-TURP changing of the prostate shape).

In order to compare dose distribution represented by analyzed indices in a reliable way, the following parameters were set equal for each patient: PTV volume, OARs volumes, number of needles and geometry of the implant. The values of parameters which have a strong influence on the work of each analyzed algorithms and consequently optimization results, were determined by preparing many plans with different variables for virtual patients' anatomy. This procedure gave us the possibility to establish planning principles, among others the values of optimization setting parameters.

The analysis of each index was done by dividing the data into three groups depending on the algorithm used in the optimization process. Statistical differences between groups were verified using $t$-test and Wilcoxon's test. Differences between groups were considered significant if the $p$-value was $<0.05$.

\section{Results}

All analyzed parameters which represent the dose (D10, D90, Dmax) are presented as percent of the prescribed dose. Those which describe the volume irradiated by the therapeutic dose (V100) and the volume covered by the $200 \%$ isodose (V200) are presented in percent of the volume. To evaluate the values of analyzed parameters, the set of data was divided into three groups according to the considered optimization method. The collected mean values of each analyzed parameter with standard deviation (SD), maximum (MAX) and minimum (MIN) values of dependent variable indices are presented in Table 1 (for prostate) and in Table 2 (for OARs). Table 3, shows the $p$ values of statistical verification of parameters analyzed in 
Table 1 The mean values (MEAN) of parameters analyzed in PTV (prostate) with standard deviation (SD), maximum (MAX) and minimum (MIN). D90 and Dmax values are presented as percentages of the prescription dose of $10 \mathrm{~Gy}$ V100 and V200 values are presented as percentages of PTV volume.

\begin{tabular}{llrrrr}
\hline Parameter & Optimization & MEAN & \multicolumn{1}{l}{ SD } & \multicolumn{1}{l}{ MIN } & \multicolumn{1}{c}{ MAX } \\
\hline Prostate & & & & & \\
D90 & GO & 75.25 & 12.19 & 53.80 & 94.49 \\
& IO & 84.51 & 12.31 & 60.63 & 101.02 \\
& BIO & 87.32 & 11.99 & 60.63 & 102.94 \\
V100 & GO & 74.79 & 7.71 & 69.97 & 86.10 \\
& IO & 81.09 & 6.85 & 70.56 & 90.76 \\
& BIO & 82.98 & 6.79 & 70.56 & 92.41 \\
V200 & GO & 12.77 & 5.76 & 5.38 & 21.41 \\
& IO & 14.24 & 6.48 & 7.91 & 32.66 \\
& BIO & 16.66 & 4.15 & 8.55 & 22.64 \\
Dmax & GO & 380.98 & 70.27 & 271.89 & 540.15 \\
& IO & 685.19 & 337.98 & 375.55 & 1815.69 \\
& BIO & 723.39 & 84.78 & 546.10 & 860.55 \\
\hline
\end{tabular}

the PTV. The corresponding $p$-values calculated between groups for parameters analyzed in OARs are shown in Table 4.

For PTV, the analysis of mean values of the dosimetric indices (D90 and V100) showed that the best results were obtained for both inverse optimization algorithms, especially BIO. The mean value of D90 for the analyzed group of patients was $84.5 \%$ for 10 and $87.3 \%$ for $\mathrm{BIO}$. The mean value of the second therapeutic index V100 was $81.1 \%$ and $83.0 \%$ for $\mathrm{IO}$ and $\mathrm{BIO}$, respectively. There was no statistically significant difference between these two groups.

Table 2 The mean values (MEAN) of parameters analyzed in OARs (urethra and rectum) with standard deviation (SD), maximum (MAX) and minimum (MIN). D10 and Dmax values are presented as percentages of the prescription dose of $10 \mathrm{~Gy}$ V100 values are presented as percentages of rectum volume.

\begin{tabular}{llrrrr}
\hline Parameter & Optimization & MEAN & \multicolumn{1}{c}{ SD } & \multicolumn{1}{c}{ MIN } & MAX \\
\hline Urethra & & & & & \\
D10 & GO & 119.85 & 11.78 & 99.77 & 138.63 \\
& IO & 125.04 & 12.51 & 111.46 & 164.96 \\
& BIO & 130.16 & 5.01 & 121.81 & 137.47 \\
Dmax & GO & 143.35 & 19.23 & 112.66 & 175.69 \\
& IO & 148.24 & 25.14 & 126.56 & 219.86 \\
& BIO & 159.53 & 27.86 & 130.47 & 243.71 \\
Rectum & & & & & \\
D10 & GO & 61.24 & 11.65 & 40.12 & 83.81 \\
& IO & 58.09 & 10.68 & 39.01 & 75.76 \\
& BIO & 57.99 & 10.69 & 38.17 & 75.77 \\
Dmax & GO & 92.75 & 26.14 & 53.68 & 141.07 \\
& IO & 85.11 & 25.03 & 53.19 & 145.26 \\
& BIO & 83.59 & 24.88 & 49.78 & 148.30 \\
V100 & GO & 0.23 & 0.50 & 0.00 & 1.69 \\
& IO & 0.08 & 0.17 & 0.00 & 0.60 \\
& BIO & 0.06 & 0.17 & 0.00 & 0.64 \\
\hline
\end{tabular}

Table 3 The $p$-values for the comparison of dosimetric parameters achieved using three different optimization methods for PTV.

\begin{tabular}{ll}
\hline Compared groups & $p$-value \\
\hline D90 - prostate & \\
GO vs. IO & 0.001 \\
GO vs. BIO & 0.000 \\
IO vs. BIO & 0.108 \\
V100 - prostate & \\
GO vs. IO & 0.002 \\
GO vs. BIO & 0.000 \\
IO vs. BIO & 0.098 \\
V200 - prostate & \\
GO vs. IO & 0.427 \\
GO vs. BIO & 0.006 \\
IO vs. BIO & 0.499 \\
Dmax - prostate & \\
GO vs. IO & 0.001 \\
GO vs. BIO & 0.000 \\
IO vs. BIO & 0.047 \\
\hline
\end{tabular}

Every $p$-value under 0.05 was marked in bold.

Better results for D90 and $\mathrm{V} 100$ and $\mathrm{BIO}$ were associated with higher values of parameters which inform about the dose escalation in the PTV. The same tendency was observed for V200 and Dmax (the highest values of doses achieved with inverse optimization algorithms, especially $\mathrm{BIO})$. For mean V200, the differences between values obtained by using different optimization algorithms were about 2\%. For Dmax, the tendency was similar, but the differences between mean values were higher. This can be additionally noticed by comparing prostate Dmax $p$-values.

Table 4 The $p$-values for the comparison of dosimetric parameters achieved using three different optimization methods for OARs.

\begin{tabular}{ll}
\hline Compared groups & $p$-value \\
\hline D1O - urethra & \\
GO vs. IO & 0.307 \\
GO vs. BIO & 0.036 \\
IO vs. BIO & 0.007 \\
Dmax - urethra & \\
GO vs. IO & 0.691 \\
GO vs. BIO & 0.017 \\
IO vs. BIO & 0.019 \\
D1O - rectum & \\
GO vs. IO & 0.002 \\
GO vs. BIO & 0.001 \\
IO vs. BIO & 0.723 \\
Dmax - rectum & \\
GO vs. IO & 0.064 \\
GO vs. BIO & 0.055 \\
IO vs. BIO & 0.153 \\
V100 - rectum & \\
GO vs. IO & 0.138 \\
GO vs. BIO & 0.138 \\
IO vs. BIO & 0.285 \\
\hline
\end{tabular}


For each statistical verification done alternately between the two groups (GO vs. IO, GO vs. BIO, IO vs. BIO) a significant difference was found ( $p$-value was in the range from 0.000 to 0.047 ).

The same situation - the best results for both parameters (V200, Dmax) obtained in plans optimized by GO, as a cost of worse D90 value - was observed in the urethra. In the second analyzed OAR, the rectum, the verification done for D10 and Dmax showed a rapid fall-off outside the implant area. Results were slightly better for BIO but the value, which differed by about $0.1 \%$ compared to IO (as was shown for D10), is not expected to be statistically different.

V100 in the rectum was analyzed to calculate the volume of the OAR (located outside the implanted area) covered by the reference isodose. As for D10 and Dmax, the lowest mean values of V100 were obtained in plans in which the BIO algorithm was used. The mean value of this parameter for 10 was approximately $33.3 \%$ higher than for $\mathrm{BIO}$ and almost four times higher for GO than for BIO. According to Wilcoxon's test, the high values of SD make these differences statistically insignificant.

To evaluate the correlation between conformity effect and optimization algorithm used, COIN was analyzed. The mean COIN values with SD for GO, IO and BIO algorithms equalled $0.655 \pm 0.100,0.756 \pm 0.096$ and $0.771 \pm 0.089$, respectively. The statistical verification proved significant differences between COIN results calculated independently between $\mathrm{GO}$ vs. IO ( $p=0.0000)$, IO vs. $\mathrm{BIO}(p=0.0342)$ and $\mathrm{GO}$ vs. $\mathrm{BIO}(p=0.0000)$.

\section{Discussion}

HDR prostate brachytherapy gives the ability to deliver highly conformal dose with rapid dose fall-off outside the implanted area [28]. As long as the positions of the needles are confirmed by performed images and monitored by measurements and quality control tests, this kind of treatment could be named intensity-modulated brachytherapy [11]. The more sophisticated algorithm is used in the TPS, the more effective reduction of doses delivered to the urethra and rectum is feasible [29]. The lowest mean values of parameters which inform about the dose escalation in the PTV (V200, Dmax) were achieved in the first group of plans, optimized with the GO algorithm. The same situation (the lowest mean values of delivered doses in plans optimized with GO) was observed in the urethra. In both cases it was positive, but on the other hand GO diminished doses delivered to the PTV (D90, V100) and thus was not satisfactory. From a clinical point of view, obtaining a required dose distribution in the PTV while minimizing doses delivered to critical organs is most important. The main therapeutic indices (D90, V100) are expected to be relatively high as they were achieved mostly by using BIO. The same applies to conformity requirements represented by COIN. COIN values were higher for BIO plans than for GO plans in every case and lower for the BIO plan than for 10 plan for one patient. As a result, BIO-COIN values were statistically different from GO-COIN and IO-COIN. Additionally, the BIO optimization algorithm worked best outside the implant area (in the rectum).

Because of normal patient anatomy, which determines that the organ at risk (urethra) is located inside the PTV, the increase of therapeutic indices for PTV (D90, V100) is connected with increasing the values of parameters analyzed in the urethra (V100, Dmax).

Statistical comparative analysis of inverse optimization (IO) and blind inverse optimization (BIO) within D90 and V100 did not show a statistically significant difference between these two groups of calculation algorithms. The mean values of D90 and V100 and IO presented in percent of the prescribed dose were respectively $2.8 \%$ and $1.9 \%$ lower than the same parameters calculated for BIO. But the parameters of descriptive statistics show an upward tendency for BIO.

The advantages of using BIO algorithms were shown in the results of statistical comparative analysis for the rectum. The analysis of D10 in the rectum revealed that outside the implant area GO does not ensure clinically desired dose gradients, although this calculation algorithm minimizes the volumes of high dose inside the implant. No statistically significant differences were found between both Dmax and V100 in the rectum and all analyzed optimization algorithms, although the mean values of parameters which describe dose distribution in this organ at risk varied by several percent. In V100, the mean value for the $\mathrm{GO}$ group was three times higher than for 10 and four times higher than for BIO.

As described in the materials and methods section, the advantage of $\mathrm{BIO}$ optimization algorithm is that it gives the opportunity to choose one of several proposed treatment plans, individually fitted to the history and treatment schedule of the patient. The other analyzed optimization algorithms implemented in the TPS do not offer such a possibility.

No matter which optimization algorithm (GO, IO or BIO) was used for calculation, manual adjustment and graphical optimization can be used to modify the final treatment plan. To make this analysis objective and to prevent the argument of the plan's being prepared in correlation with physicist's experience, these subjective modifying tools were not used.

3D-CBRT may be used for local increase of the dose (boost). In this case, the technique involving higher doses applied in some areas in the prostate by increasing the high-dose volume around one or more needles is called the 'boost-in-boost strategy', as it is unaffected by organ motion, PTV volume changes and setup errors $[5,17,20]$.

\section{Conclusions}

Application of the blind inverse optimization (BIO) algorithm led to clinically best dose indices for PTV and the urethra, as confirmed by the values of the therapeutic indices in PTV (D90, V100) and tracked dose parameters for the rectum. Application of geometrical optimization (GO) led to smaller doses in the urethra, which was however associated with certain dose decrease also in PTV, as confirmed by the values of all studied indices.

\section{References}

[1] Chicheł A, Skowronek J, Kanikowski M. Thermal boost combined with interstitial brachytherapy in breast conserving therapy - assessment of early toxicity. Rep Pract Oncol Radiother 2011;16:87-94. 
[2] Palvolgyi J. Influence of different Fletcher-Suit applicator geometries on sagittal dose distribution. Phys Med 2010;26(1): 49-54.

[3] Polgár C, Jánváry L, Major T, Somogyi A, Takácsi-Nagy Z, Fröhlich $G$, et al. The role of high-dose-rate brachytherapy boost in breast-conserving therapy: long-term results of the Hungarian National Institute of Oncology. Rep Pract Oncol Radiother 2010;15:1-7.

[4] Shah AP, Strauss JB, Kirk MC, Chen SS, Dickler A. A dosimetric analysis comparing electron beam with the MammoSite brachytherapy applicator for intact breast boost. Phys Med 2010; 26(2):80-7.

[5] Kovács G, Pötter R, Loch T, Hammer J, Kolkman-Deurloo IK, de la Rosette JJMCH, et al. GEC/ESTRO-EAU recommendations on temporary brachytherapy using stepping sources for localised prostate cancer. Radiother Oncol 2005;74:137-48.

[6] Sullivan L, Williams SG, Tai KH, Foroudi F, Cleeve L, Duchesne GM. Urethral stricture following high dose rate brachytherapy for prostate cancer. Radiother Oncol 2009;91: 232-6.

[7] Pieters BR, van de Kamer JB, van Herten YRJ, van Wieringen $N$, D'Olieslager $G M$, van der Heide $U$, et al. Comparison of biologically equivalent dose-volume parameters for the treatment of prostate cancer with concomitant boost IMRT versus IMRT combined with brachytherapy. Radiother Oncol 2008;88:46-52.

[8] Sandhu GK, Dunscombe PB, Khan RFH. A pre-clinical phantom comparison of tissue harmonic and brightness mode imaging for application in ultrasound guided prostate brachytherapy. Phys Med 2011;27(3):153-62.

[9] Mocna M, Zwierzchowski G. Dosimetric verification of the dose calculation algorithms in real time prostate brachytherapy. Rep Pract Oncol Radiother 2008;13:275-9.

[10] Camgöz B, Yeğin G, Kumru MN. Differential dose contributions on total dose distribution of ${ }^{125} \mathrm{I}$ brachytherapy source. Rep Pract Oncol Radiother 2010;15:69-74.

[11] Demanes DJ, Rodriguez RR, Schour L, Brandr D, Altieri G. High-dose-rate intensity-modulated brachytherapy with external beam radiotherapy for prostate cancer: California endocurietherapy's 10-year results. Int J Radiat Oncol Biol Phys 2005;61:1306-16.

[12] Kim Y, Hsu ICJ, Lessard E, Kurhanewicz J, Noworolski SM, Poulior J. Class solution in inverse planned HDR prostate brachytherapy for dose escalation of DIL defined by combined MRI/MRSI. Radiother Oncol 2008;88:148-55.

[13] Toossi MTB, Abdollahi M, Ghorbani M. Monte Carlo dose calculation of GZP6 ${ }^{60} \mathrm{Co}$ stepping source based on a matrix shift technique. Rep Pract Oncol Radiother 2011; $16: 10-3$.

[14] Bachand F, Martin AG, Beaulieu L, Harel F, Vigneault E. An eight-year experience of HDR brachytherapy boost for localized prostate cancer: biopsy and PSA outcome. Int J Radiat Oncol Biol Phys 2009;73:679-84.

[15] Kolkman-Deurloo IKK, Deleye XGJ, Jansen PP, Koper CM. Anatomy based inverse planning in HDR prostate brachytherapy. Radiother Oncol 2004;73:73-7.

[16] Astrom L, Pedersen D, Mercke C, Holmang S, Johansson K. Long-term outcome of high dose rate brachytherapy in radiotherapy of localize prostate cancer. Radiother Oncol 2005;74:157-61.

[17] Martin T, Röddiger S, Kurek R, Dannenberg T, Eckart $O$, Kolotas $\mathrm{C}$, et al. 3D conformal HDR brachytherapy and external beam irradiation combined with temporary androgen deprivation in the treatment of localized prostate cancer. Radiother Oncol 2004;71:35-41.

[18] Uniyal SC, Sharma SD, Naithani UC. A dosimetry method in the transverse plane of HDR Ir-192 brachytherapy source using gafchromic EBT2 film. Phys Med; 2011. doi: 10.1016/j.ejmp.2011.03.005.

[19] Slosarek K, Bystrzycka J, Fijałkowski M. Real time brachytherapy for prostate cancer - a new challenge for medical physicists. Rep Pract Oncol Radiother 2005;10:255-9.

[20] Vicini F, Vargas C, Edmundson G, Kestin L, Martinez A. The role of high dose rate brachytherapy in locally advanced prostate cancer. Sem Rad Oncol 2003;13:98-108.

[21] Hoskin P, Motohashi K, Bownes P, Bryant L, Ostler P. High dose rate brachytherapy in combination with external beam radiotherapy in the radical treatment of prostate cancer: initial results of a randomised phase three trial. Radiother Oncol 2007;84:114-20.

[22] Ghadjar P, Matzinger O, Isaak B, Behrensmeier F, Stroux A, Rentsch C, et al. Accociation of urethral toxicity with dose exposure in combined high-dose-rate brachytherapy and intensity-modulated radiation therapy in intermediate- and high-risk prostate cancer. Radiother Oncol 2009;91:237-42.

[23] Akimoto T, Katoh H, Kitamoto Y, Shirai K, Shioya M, Nakano T. Anatomy-based inverse optimization in high-dose-rate brachytherapy combined with hypofractionated external beam radiotherapy for localized prostate cancer: comparison of incidence of acute genitourinary toxicity between anatomybased inverse optimization and geometric optimization. Int J Radiat Oncol Biol Phys 2006;64:1360-6.

[24] Hoskin P, Bownes P, Ostler P, Walken K, Bryant L. High dose rate afterloading brachytherapy for prostate cancer: catheter and gland movement between fractions. Radiother Oncol 2003;68:285-8.

[25] Kirisits C, Siebert FA, Baltas D, De Brabandere M, Hellebust TP, Berger D, et al. Accuracy of volume and DVH parameters determined with different brachytherapy treatment planning systems. Radiother Oncol 2007;84:290-7.

[26] Lahanas M, Baltas D, Zamboglou N. Anatomy-based threedimensional dose optimization in brachytherapy using multiobjective genetic algorithms. Med Phys 1999;26(9):1904-18.

[27] Baltas D. A handbook for the optimization and optimization tools in SWIFT Version 3.0 (Oncentra Prostate), SWIFT\&Oncentra Prostate Users Meeting, Kraków 2007.

[28] Yoshioka Y, Nishimura T, Kamata M, Harada H, Kanazawa K, Fuji $\mathrm{H}$, et al. Evaluation of anatomy-based dwell position and inverse optimization in high-dose-rate brachytherapy of prostate cancer: a dosimetric comparison to a conventional cylindrical dwell position, geometric optimization, and dosepoint optimization. Radiother Oncol 2005;75:311-7.

[29] Yoshioka Y, Konishi K, Oh RJ, Sumida I, Yamazaki H, Nakamura $S$, et al. High dose rate brachytherapy without external beam irradiation for locally advanced prostate cancer. Radiother Oncol 2006;80:62-8. 